\title{
Evidence of Cerebellar Dysfunction in Children with Myelomeningocele
}

\author{
Martin $\mathbf{S}^{1 *}$ and Kitzman $\mathbf{P}^{2}$ \\ ${ }^{1}$ Department of Physical Therapy, College of Education \\ and Health Sciences, University of Evansville, USA \\ ${ }^{2}$ Department of Rehabilitation Sciences, University of \\ Kentucky, USA \\ *Corresponding author: Suzanne Martin, Department \\ of Physical Therapy, University of Evansville, 1800 \\ Lincoln Avenue, Evansville, USA
}

Received: J anuary 21, 2017; Accepted: February 13, 2017; Published: February 15, 2017

\begin{abstract}
Structural differences in the development of the cerebellum in children with spina bifida myelomeningocele are associated with functional deficits in movement, timing, and attention. Furthermore, these structural and functional deficits contribute to uneven development of perception and cognition. Studies are reviewed that provide some understanding of the reason such a mixed pattern of assets and deficits within the same area is seen in these children. Motor learning and adaptation are relative strengths while predictive motor control is not. Upper limb function and visual perception are problematic as is timing and attention. In utero damage to the cerebellum may provide an explanation for this mixed pattern. Early damage can negatively impact the development of cerebellar circuits which could interfere with learning. Clinicians need to be aware of the relative strengths and weaknesses in children with spina bifida myelomeningocele in order to individualize early intervention treatment plans, optimize motor learning, and promote maximal participation in daily life.
\end{abstract}

Keywords: Cerebellar Dysfunction; Spina Bifida; Myelomeningocele; Perception; Motor Learning

\section{Introduction}

Spina bifida (SB) is one of the most debilitating birth defects affecting the central nervous system with a prevalence of 3 in 10,000 live births [1]. While the prevalence has declined since the implementation of folic acid fortification, 1500 babies are born with spina bifida annually in the US. Spina bifida myelomeningocele (SBM) is the most severe and common form of SB. It is characterized by failure of neural tube development and atypical brain development [2]. The congenital defect consists of a cystic protrusion of the spinal cord through open vertebral arches resulting in motor paralysis and sensory loss below the level of the spinal lesion. Children born with this defect experience limited mobility secondary to paralysis of the lower extremities. Congenital malformations of the cerebellum, corpus callosum, and the midbrain commonly occur in SBM that further disrupt brain development [3].

Children with SBM present with a mixed pattern of strengths and weaknesses often within the same developmental areas. Sensory loss and motor paralysis of the trunk and lower extremities is dependent on the level of the spinal lesion. Mobility is impaired which affects the ability of the child to explore the environment. The child may need orthoses and an assistive device to walk. Major functional deficits are seen in movement, timing, and attention. Upper extremity impairments have also been recognized clinically for some time but have not been explained based on the underlying pathology of SBM [4-6]. Damage to the developing cerebellum could disrupt the establishment of the cortico-cerebellar circuits that support procedural learning, modulation of attention, and social skill acquisition. Understanding the functional deficits associated with the cerebellar malformations seen in SBM could assist in guiding early intervention strategies.
The cerebellum in children with SBM is structurally different from a typically developing child's cerebellum. Some parts of the cerebellum are smaller and some parts are larger. Children with SBM exhibit significant reductions in cerebellum volumeas well as structural deficits $[7,8]$. The volume of the anterior lobe of the cerebellum is increased while the posterior lobe of the cerebellum is decreased. The anterior lobe is involved in motor function while the posterior lobe is part of the cognitive cerebellum. The cerebellum regulates motor output by comparing sensory information from the intended movement with the sensory information from the actual movement. Early motor learning depends on the cortico-striatal and cortico-cerebellar systems. In later motor learning only one of these systems is needed to consolidate and maintain memory for the new motor sequence.

The majority of children with SBM also have an Arnold-Chiari-II malformation (ACM), and exhibit hydrocephalus requiring placement of a ventriculoperitoneal shunt. ACM is a cranial malformation of the posterior cranial fossa. A small posterior fossa restricts the development of the cerebellum. In ACM, the size of the cerebellar hemispheres is reduced, causing the cerebellar vermis to be displaced downward, shifting the medulla and cervical cord downward and elongating the medulla [9]. Additional changes include tectal beaking (structural changes in the roof of the midbrain) and wrapping of the cerebellar hemispheres around the brainstem [10]. The size of the posterior fossa and consequently the volume of the cerebellum are reduced in children with SBM. Moreover, the reductions are not merely a linear down scaling. The anterior cerebellum enlarges while the posterior cerebellum is reduced. Cerebellar pathology plays a central role in causing the motor and perceptual findings in children with SBM [11].

The cerebellum is an important part of the neural network for 
procedural motor learning [12]. The anterior lobe of the cerebellum is intact and enlarged in children with SBM while the posterior lobe of the cerebellum is reduced [13]. The reduction of the posterior cerebellum produces deficits in the cognitive part of the cerebellum. The cognitive cerebellum involves spatial processing and visual working memory [14]. Changes in structure and underdevelopment of the cerebellum are prominent features in the neuropathology of SBM [2].

Cerebellar dysfunction has been documented in a wide variety of neurodevelopmental disorders ranging from autism spectrum disorder (ASD) [15], attention deficit hyperactive disorder (ADHD) to Joubert syndrome [16], a rare genetic disorder. Recent reviews have linked the cognitive, language, and social deficits exhibited by these children to cerebellar malformations $[17,18]$. Abnormalities of the cerebellum are routinely described in studies of children with ASD $[19,20]$ and symptom severity has been correlated with a reduction in cerebellar volume [21]. Decreases in cerebellar gray matter have also been documented in different cerebellar sub regions depending on the neurodevelopmental disorder described.

\section{Functional Deficits}

Functional deficits are those that are always present in children with SBM and are apparent in infancy; persist in childhood, adolescence and adulthood. The three functional deficits are movement, timing and attention. All have been linked to the CNS damage that occurs with SBM [22]. They are all weakly correlated to each other but are definitely associated with structural changes in the brain. The variation in the magnitude of these functional deficits is thought to be related to the degree of structural changes in the cerebellum caused by ACM $[11,23]$. Because of the variation in functional deficits, children with SBM present with a mixed pattern of strengths and weaknesses often within the same areas. A summary of reviewed studies can be found in Table 1.

\section{Movement}

Impaired lower extremity movement is related to the level of the spinal lesion in children with SBM. Children with higher lesions have more movement dysfunction than children with lower lesions. The spinal cord may or may not be intact and functional below the lesion level [24]. Lower levels of motor performance were found to be predicted by having a higher lesion level, that is, more of the spinal cord involved or shunted hydrocephalus than by a lower lesion level and no shunt [25]. The spinal cord deficits and subsequent participation restriction caused by impaired mobility may contribute to delayed sensorimotor development. Cerebellar volume and structural changes related to ACM affect motor regulation and sensory-motor timing in children with SBM [23].

Two-thirds of children with SBM exhibit impaired upper limb function which can be linked to cerebellar dysmorphology [26]. Children with SBM demonstrate upper extremity weakness, hand weakness, poor hand function, and impaired kinesthetic awareness [4-6,27]. Young adults with SBM demonstrated deficits on four tests of upper extremity function: arm posture, rebound, finger to nose (dysmetria) and rapid alternating movements of the forearm (dysdiadochokiness) [26]. Very few subjects achieve perfect scores on the rebound and arm posture tasks whereas the control group obtained perfect scores. Group differences were not related to age at the time of testing, complications, lesion group or number of shunt revisions. However, both the SBM group and their age-matched peers demonstrated similar decrements in performance when the coordination tests were accompanied by an additional physical or cognitive demand such as closing the eyes or counting backwards from 50 at the same time. The SBM group had more difficulty with the cognitive challenge during the finger to nose to finger task than the control group.

Upper limb function was further studied by Jewell and colleagues [28] in an attempt to quantify the effect of lesion level on independent function. The Scales of Independent Behavior- Revised (SIB-R) were used to measure function in four domains: personal living, community living, social/communication and motor. The only upper limb test that significantly correlated with all domains was rebound performance. Timing deficits were seen in two of the upper extremity tests, diadochokinesis and limb dysmetria. Children with higher lesions took longer to perform the dysmetria task. Findings from a previous study [26] also suggested that the level of independent functioning in children with SBM may be compromised by upper limb impairments.

Other studies of independence in this population have focused on activities of daily living. Peny-Dahlstrand and fellow researchers [29] concluded that children with SBM need help to learn how to do things and also how to get things done. Motor planning was difficult as was generating the right amount of force during movements. Children with SBM were less able to respond to relevant cues in the environment to complete tasks.

Motor learning and error-based motor adaptation are relative strengths for children with SBM $[23,30,31]$. While Lomax-Bream and associates [25] found slower rates of growth in language and cognition as part of a longitudinal study of young children with SBM, these same children had accelerated growth in motor skills. Studies have shown that children with SBM can learn motor tasks such as moving a lever quickly [32] or drawing a mirror image [30]. The fact that the volume of the anterior cerebellum is not decreased but actually increased in children with SBM may explain the preservation of motor learning in this population. However, reduced volume in the posterior cerebellum has been linked to difficulties in motor control. Children with SBM made more errors than controls while learning a sequence of movements to draw a mirror image [30]. The lack of predictive, dynamic motor control may be due to the inability of the child with SBM to produce an internally generated template for movement. Hand function is impaired when performing daily tasks such as turning pages, eating, and stacking checkers [4,32]. Deficits in temporal motor regulation could explain the problems with gross and fine motor control.

\section{Timing}

Children with SBM have difficulty perceiving and producing timing and rhythm. Researchers compared perceptual timing and motor timing in children with and without SBM [33]. Children with SBM had significantly more difficulty than age-matched controls on judging the duration of a timing task of $400 \mathrm{~ms}$ duration. Children with SBM performed as well as the control group when judging frequency of around $3000 \mathrm{~Hz}$. This indicates that perceptual timing problems 
Table 1: Studies of Functional and Perceptual/Cognitive Deficits in Children with SBM.

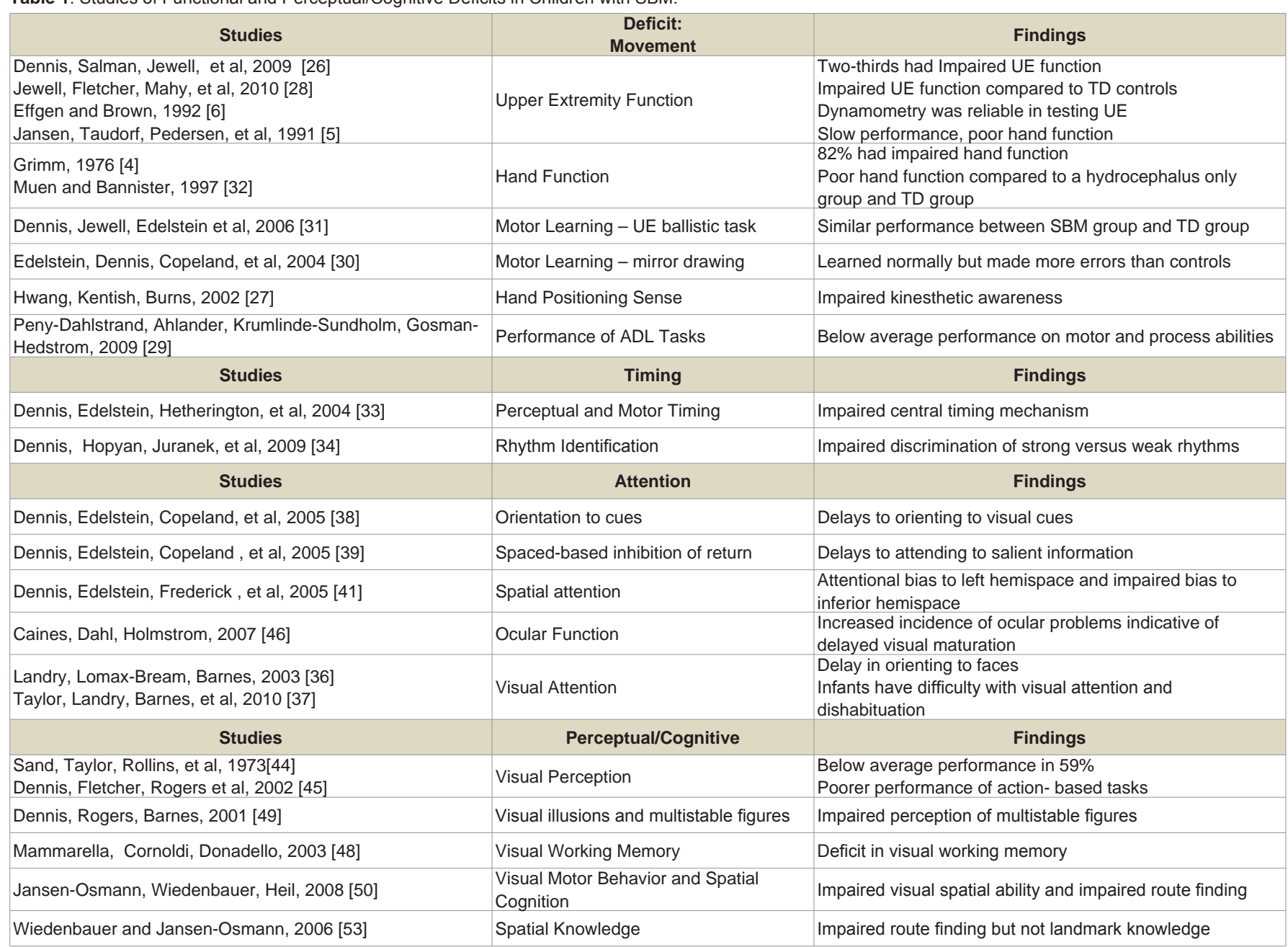

SBM: Spina Bifida Myelomeningocele; TD: Typically Developing; UE: Upper Extremity; ADL: Activity of Daily Living.

noted in the children with SBM are probably not due to any general hearing deficit. Furthermore, the researchers related the children's abilities on the timing tasks to their cerebellar volume measurements. Perception of the duration of a timing task was negatively correlated with cerebellar volume in the typically developing group while it was positively correlated with cerebellar volume in the SBM group.

Impairments of central timing that may be the result of poor development of the cerebellum. The anterior and posterior lobes of the cerebellum are involved in producing structured and unstructured rhythms and both of these cerebellar regions are abnormal in SBM [34]. Children with SBM demonstrate deficits on motor timing tasks such as tapping when the task is unpaced but not when it is paced by an external cue. Tapping is a measure of motor timing rather than perceptual timing. There are two components to a tapping task, a clock component that represents a central timekeeper and a motor component that represents the motor implementation [35]. Each component can contribute to the variance of the rhythmic tapping task. Children with SBM are better at producing rhythms that are externally cued such as from a metronome rather than from an internally generated model because the internal timing signal is faulty. Children with SBM showed more variance in the clock component of the task than the control group. The number of valid tapping trials was positively correlated with anterior and inferior-posterior cerebellar volumes in the children with SBM. Even though perceptual and motor timing are different and controlled by separate structures, these two components are related to each other as evidenced by a fair level of correlation [33]. This may indicate that children with SBM have a central timing impairment.

\section{Attention}

Attention is the third functional deficit demonstrated by children with SBM [22]. Children need to attend to salient features within their environment to learn about the world. The deficits in attention in children with SBM are linked to structural changes in the roof of the midbrain and the posterior cortex, not the cerebellum directly. However, because the cerebellum uses sensorimotor experiences to form internal representations that can be used to predict motor consequences, the deficits in attention will impact the ability of the child with SBM to learn from exploration. Visual attention was shown to be impaired in infants with SBMbased on a delay in orienting to faces [36]. Eighteen month old infants with SBM have difficulty with visual attentions and dishabituation [37]. The problem continues in older children who have difficulty orienting to external stimuli and then, once engaged, terminating their focus [38]. 
Dennis and colleagues [39] compared the ability of typically developing children with that of children with SBM in performing a test of peripersonal spatial attention. Peripersonal space is the area within arm's reach used for activities like picking up objects, drawing or bisecting lines [40]. Peripersonal space development is dysfunctional in children with SBM as evidenced by frequent errors in bisecting lines. Furthermore, children with SBM have a preference for looking and attending to left hemispace and to inferior hemispace [41]. Spatial biases have been noted as both a cause and a result of poor motor regulation [11]. Visual attention and function is crucial for later development of cognitive skills in children. Visual processing abilities in children with and without SBM have been found to predict later cognitive ability [36].

\section{Perception and Cognition}

The three functional deficits of movement, timing, and attention present in children with SBM negatively impact their perceptual and cognitive development. While the majority of children with SBM have a low normal IQ, sensorimotor deficits combined with structural changes in the cerebellum can interrupt the development of perception and cognition. Furthermore, children with SBM often appear less motivated to master their environment and to develop goal-directed behavior.

The cerebellum's distinct role in motor sequence learning has been established [12,42]. The typically developing cerebellum produces internal models of movement which support the rapid skill acquisition that occurs early in human development. Once these models are developed the cerebellum regulates motor output by comparing sensory information from the intended movement with the sensory information from the movement that actually occurred [43]. The cerebellum detects errors if the movement deviates from the intended movement and makes corrections [43]. It also regulates postural tone throughout the body to support movement execution. Damage to the cerebellum during the development of the circuits connecting the cerebellum to other cerebral cortical areas could interfere with implicit or procedural learning [17].

Visual perceptual deficits have been documented in children with SBM [44]. The cerebellum, posterior cortex and the dorsal stream pathways are utilized in action-based tasks and spatial cognition. Dennis, Fletcher, Rogers, Hetherington, and Francis [45] studied visual perception in children with spina bifida and hydrocephalus (SBH). The children with SBH had no significant intellectual impairment. The study found bigger differences in performance between the SBH group and controls when the visual perceptual task was action-based rather than object-based. They hypothesized that the reason for this difference was that the children with SBH use ventral stream visual processing more easily than dorsal stream processing. The ventral stream is the neural pathway for object vision. It has been called the "what" system because the object's form, color, texture and size are recognized. The dorsal stream is the spatial system or the "where" system [43]. Action-based tasks include stereopsis, mental rotation, and figure-ground perception. Stereopsis refers to the quality of visual fusion. This deficit may reflect oculomotor impairments in the brainstem which could impair stereoacuity. Caines, Dahl, and Holmstrom [46] reported a high incidence of ocular disturbances in a population-based study of children with SBM. Object-based tasks such as facial recognition and object identification were performed better than action-based tasks by children with SBM. Goal directed actions in play have been reported to be decreased in children with SBM [47] but not as yet specifically linked to difficulties with actionbased tasks.

Mammarella, Cornoldi and Donadello [48] studied visuospatial working memory in children with SBM. Children with SBM had difficulty visualizing and remembering actions they were to solve mentally. They were given a battery of tests, one provided information about visual working memory separate from visuospatial working memory. The 'House Visual Span' test measured visual working memory. The children with SBM and the age-matched controls performed similarly on the visuospatial working memory tests. Only children with SBM showed impairment on the 'House Visual Span' test.

Children with SBM also have difficulty constructing a situational model of space $[49,50]$. The problem stems from the child's prominent figure-ground perceptual deficit and in not perceiving visual illusions that change over time (perceptual multistability) [49]. This later perceptual ability requires feedback from the frontal and parietal areas of the brain to the visual cortex, a top-down feedback system that is impaired in children with SB $[49,51]$.

Researchers [50] studied how much visual motor behavior and active motor exploration was needed for spatial cognition in a group of children with SBM. Spatial cognition is defined as "higherorder visual spatial abilities involving the ability to recognize and remember the relationships between features within an object or design, between two or more objects, or between oneself and objects" [52]. In concurrence with Dennis and colleagues [45], the children in Jansen-Osmann's study [50] also demonstrated impairments on tasks that required dorsal stream visuospatial processing. The differences between the experimental and control groups disappeared when performance IQ was taken into account. The authors linked the performance on visuospatial tasks in a small-scale space to nonverbal intelligence. However, the SBM group performed less well than the control group on a virtual maze, a large-scale space. The authors based their explanation on the diminished level of mobility demonstrated by the SBM group since the cognitive differences were controlled for between the groups. An earlier study demonstrated impaired route finding but not impaired knowledge of landmarks in children with SBM compared to healthy control children [53]. This finding supports lack of mobility as a potential causative factor in decreased spatial cognition in this group of children.

The underlying deficit in children with SBM appears to be a processing bias toward associative processing rather than assembled processing [22]. This processing dichotomy can be applied to motor function, perceptual function, and language function and goes a long way to begin to unravel the puzzle of strengths and weaknesses seen in children with SBM. Associative processing is the ability to form associations, modifications, and categorizations [23]. Associative processing would allow for motor adaptation, perception of categories and interpersonal communication. This fact would explain the motor learning piece.

Assembled processing is described as an on-line process of acting and disengaging while creating feed-forward models to guide future 
performance. Deficits in assembled processing would manifest as problems with anticipatory control which are seen in this group of children. Furthermore, coordinate perception is impaired as exemplified by poor spatial route finding, decreased figure-ground perception, poor stereopsis, and decreased visual form constancy. In the language area, children with SBM have difficulty with contextual meanings, understanding social scripts and making inferences, all of which represent assembled processing. This goes a long way to explaining the problem of putting it all together.

Impairment of executive function, a specific form of cognition, is seen in children with SBM. Skills related to executive function involve planning and executing tasks. Executive function connects past and present actions mentally. Attention is required, one of the functional deficits in this group of children. Additionally, inhibiting competing responses and pacing work is required, both of which may be difficult for the child with SBM. Despite the finding of poor executive function on psychological testing, [54] children with SBM do not exhibit global deficits in this area [23]. Other researchers found that when the visual-motor and cognitive requirements were removed by using computerized tasks of attention, the differences in scores of attention were no longer significant [55]. Sustained attention is relatively intact but problem solving is slower as is performance on typical tests of executive function.

\section{Implications for Practice}

Clinically, children with SBM present with a mixed pattern of assets and deficits often within the same area. For example, adaptive movement is a relative strength, while predictive movement is a weakness; feature detection is a strength while visually guided goaldirected actions are a weakness. Adaptive movement involves implicit learning as a result of repeated exposure to a task or unconscious practice, learning without intent. The cerebellum is an important part of the neural network for procedural motor learning [12]. The anterior lobe of the cerebellum is intact and enlarged in children with SBM while the posterior lobe of the cerebellum is reduced [13]. The reduction produces deficits in the cognitive part of the cerebellum. The cognitive cerebellum involves spatial processing and visual working memory [14]. The ability to learn in the presence of changing task demands that require action or modification of action is impaired in this group of children.

Dynamic movements, orienting to external cues, demonstrating coordinate perception, use of timing for motor regulation and the ability to perform self-care tasks efficiently are all problematic for the child with SBM and should therefore be the focus of intervention. Cerebellar damage produces functional deficits as well as perceptual and cognitive deficits in children with SBM. These deficits reflect poor feed-forward control in movement, learning, and play. Informing and educating parents about the potential lack of congruence between their child's capacity for motor learning and their child's motor performance may help reinforce autonomy in their child from a young age. Movement goals should not only focus on motor sequence learning but on timed performance and utilization of environmental cues to guide movement adaptation. Pretend play is one way to encourage development of language and social skills while utilizing movement to drive cognition.

\section{Summary}

Abnormal structural development of the cerebellum in children with SBM impacts their function and results in a complex pattern of strengths and weaknesses. Functional deficits are seen in movement, timing, and attention. Paralysis of the lower extremities is evident and related to the level of the developmental defect. Two-thirds of children with SBM also exhibit upper extremity impairments due to cerebellar structural changes $[13,26]$. Despite the ability to learn motor tasks, the ability to adapt tasks while learning a sequence of movements is impaired in this group of children. The significance of upper limb dysfunction is seen in its impact on independent function and quality of life. Upper limb impairments, visual perceptual and spatial perceptual deficits in children with SBM have been recognized clinically for some time and can now be explained based on cerebellar pathology. Research should be focused on interventions that would foster visual spatial skills, attention, and timing as well as motor learning within the context of independent participation in all aspects of life.

\section{References}

1. Center for Disease Control. 2016.

2. Barkovitch J. Pediatric Neuroimaging. Philadelphia PA: Lippincott, Williams \& Wilkins, 2005.

3. Fletcher JM, Francis DJ, Thompson NM, Davidson KC, Miner ME. Verbal and nonverbal skill discrepancies in hydrocephalic children. J Clin Exper Neuropsychol. 1992; 14: 593-609.

4. Grimm RA. Hand function and tactile perception in a sample of children with myelomeningocele. Am J Occup Ther. 1976; 30: 234-240.

5. Jansen J, Taudorf K, Pedersen H, Jensen K, Seitzberg A, Smith T. Upper extremity function in spinabifida. Child's Nerv Syst.1991; 7: 67-71.

6. Effgen SK, Brown DA. Long-term stability of hand-held dynamometric measurements in children who have myelomeningocele. Phys Ther. 1992; 72: 458-465.

7. Fletcher JM, Bohan TP, Brandi ME, Kramer LA, Brookshire BL, Thorstad K, et al. Morphometric evaluation of the hydrocephalic brain: relationships with cognitive abilities. Childs Nerv Syst. 1996; 2: 192-199.

8. Juranek J, Dennis M, Cirino PT, El-Messidi L, Fletcher JM. The cerebellum in children with spina bifida and Chiari II malformation: quantitative volumetrics by region. Cerebellum. 2010; 9: 240-248.

9. Oaks W, Gaskill S. Symptomatic chiari malformations in childhood. Park TS, editor. In: Spinal Dysraphism. Blackwell Science Inc. 1992; 104-125.

10. Juranek J, Salman MS. Anomalous development of brain structure and function in spina bifida myelomeningocele. Dev Disabil Res Rev. 2010; 16: 23-30.

11. Dennis M, Salman MS, Juranek J, Fletcher JM. Cerebellar motor function in spina bifida meningomyelocele. Cerebellum. 2010; 9: 484-498.

12. Doyon J, Benali H. Reorganization and plasticity in the adult brain during learning of motor skills. Cur OpinNeurobiol. 2005; 15: 161-167.

13. Dennis M, Spiegler BJ, Simic N, Sinopoli KJ, Wilkinson A, Yeates KO, et al. Functional brain plasticity in childhood braindisorders: When, what, how and whom to assess. Neuropsychol Rev.2014; 42: 389-408.

14. Stoodley CJ. The cerebellum and cognition: Evidence from functional imaging studies. Cerebellum. 2012; 11: 352-365.

15. Courchesne E. Brain development in autism: early overgrowth followed by premature arrest of growth. Men Ret Dev Dis. 2004; 10: 106-111.

16. Tavano A, Borgatti R. Evidence for a link among cognition, language and emotion in cerebellar malformations. Cortex. 2010; 46: 907-918. 
17. Stoodley CJ. The cerebellum and neurodevelopmental disorders. Cerebellum 2016; 15: 34-37.

18. Stoodley CJ, Limperopoulos C. Structure-function relationships in the developing cerebellum: evidence from early-life cerebellar injury and neurodevelopmental disorders. Semin Fetal Neonatal Med. 2016; 21: 356 364.

19. Wang SS, Kloth AD, Badura A. The cerebellum, sensitive periods, and autism. Neuron. 2014; 83: 518-532.

20. Becker EB, Stoodley CJ. Autism spectrum disorder and the cerebellum. Int Rev Neurobiol. 2013; 113: 1-34.

21. Castellanos F, Lee P, Sharp W, Jeffries N, Greenstein D, Clasen L, et al. Developmental trajectories of brain volume abnormalities in children and adolescents with attention-deficit/hyperactivity disorder. JAMA. 2002; 288 1740-1748.

22. Dennis M, Landry SH, Barnes M, Fletcher JM. A model of neurocognitive functions in spina bifida over the life span. J Inter Neuropsychol Soc. 2006 12: $285-296$.

23. Dennis M, Barnes MA. The cognitive phenotype of spina bifida meningomyelocele. Dev Dis Res Rev. 2010; 16: 31-39.

24. Lomax-Bream LE, Barnes M, Copeland K, Taylor HB, Landry SH. The impac of spina bifida on development across the first 3 years. Dev Neuropsychol. 2007; 31: 1-20

25. Stark GD, Baker GCW. The neurological involvement of the lower limbs in myelomeningocele. Dev Med Child Neurol. 1967; 9: 732-744.

26. Dennis M, Salman S, Jewell D, Hetherington R, Spiegler BJ, MacGregor $\mathrm{DL}$, et al. Upper limb motor function in young adults with spina bifida and hydrocephalus. Childs Nerv Syst. 2009; 25: 1447-1453.

27. Hwang R, Kentish M, Burns $Y$. Hand positioning sense in children with spina bifida myelomeningocele. Aust J Physio. 2002; 48: 17-22.

28. Jewell D, Fletcher JM, Mahy CEV, Hetherington R, MacGregor D, Drake JM, et al. Upper limb cerebellar motor function in children with spina bifida. Childs Nerv Syst. 2010; 26: 67-73.

29. Peny-Dahlstrand $M$, Ahlander AC, Krumlinde-Sundholm L, GosmanHedstrom G. Quality of performance of everyday activities in children with spina bifida: a population-based study. Acta Paediatrica. 2009; 8: 1674-1679.

30. Edelstein K, Dennis M, Copeland K, Frederick J, Francis D, Hetherington $\mathrm{R}$, et al. Motor learning in children with spina bifida: Dissociation between performance level and acquisition rate. J Inter Neuropsychol Soc. 2004; 10 877-887.

31. Dennis M, Jewell D, Edelstein K, Brandt ME, Hetherington R, Blaser SE, et al. Motor learning in children with spinabifida:intact learning and performance on a ballistic task. J Int Neuropsychol Soc. 2006; 12: 598-608.

32. Muen, WJ, Bannister CM. Hand function in subjects with spina bifida. Eur $J$ Pediatr Surg. 1997; 7: 18-22.

33. Dennis M, Edelstein K, Hetherington R, Copeland K, Frederick J, Blaser SE, et al. Neurobiology of perceptual and motor timing in children with spina bifida in relation to cerebellar volume. Brain. 2004; 127: 1292-1301.

34. Dennis M, Hopyan T, Juranek J, Cirino PT, Hasan KM, Fletcher J. Strong meter and weak-meter rhythm identification in spina bifida meningomyelocele and volumetric parcellation of rhythm-relevant cerebellar regions. Ann NY Acad Sci. 2009; 1169: 84-88.

35. Wing AM, Kristofferson AB. Response delays and the timing of discrete motor responses. Percep Psychophys. 1973; 14: 5-12.

36. Landry SH, Lomax-Bream L, Barnes M. The importance of early motor and visual functioning for later cognitive skills in preschoolers with and without spina bifida. J Int Neuropsychol Soc. 2003; 9: 175.

37. Taylor HB, Landry SH, Barnes M, Swank P, Cohen LB, Fletcher J. Early information processing among infants with and without spina bifida. Inf Beh Dev. 2010; 33: 365-372

38. Dennis M, Edlestein K, Copeland K, Frederick J, Francis DJ, Hetherington R, et al. Covert orienting to exogenous and endogenous cues in children with spina bifida. Neuropsychologia. 2005; 43: 976-987.

39. Dennis M, Edelstein K, Copeland K, Frederick J, Francis DJ, Hetherington $\mathrm{R}$, et al. Space-based inhibition of return in children with spina bifida. Neuropsychol. 2005; 19: 456-465.

40. Halligan PW, Fink GR, Marshall JC, Vallar G. Spatial cognition: evidence from visual neglect. Trends Cog Sci. 2003; 7: 125-133.

41. Dennis M, Edelstein K, Frederick J, Copeland K, Francis DJ, Blaser SE, et al. Peripersonal spatial attention in children with spina bifida: Associations between horizontal and vertical line bisection and congenital malformations of the corpus callous, midbrain, and posterior cortex. Neuropsycholog. 2005; 43: $2000-2010$.

42. Orban $\mathrm{P}$, Peigneux $\mathrm{P}$, Lungu $\mathrm{O}$, Albouy $\mathrm{G}$, Breton $\mathrm{E}$, Laberenne $\mathrm{F}$, et al. The multifaceted nature of the relationship between performance and brain activity in motor sequence learning. Neuro Image. 2010; 49: 694-702.

43. Kandel ER, Schwartz JH. Principles of Neural Science, $5^{\text {th }}$ ed. New York, NY McGraw-Hill Medical, 2013.

44. Sand P, Taylor N, Rawlings M, Chitnis S. Performance of children with spina bifida manifesta on the Frostig Developmental Test of Visual Perception. Percep Mot Skills.1973; 37: 539-546.

45. Dennis J, Fletcher JM, Rogers T, Hetherington R, Francis DJ. Objectbased and action-based visual perception in children with spina bifida and hyddrocehalus. J Int Neuropsych. 2002: 8: 95-106.

46. Caines E, Dahl M, Holmstrom, G. Longterm oculomotor and visual function in spinal bifida cystica: a population study. Acta Ophthalmol Scand. 2007; 85: 662-666.

47. Landry SH, Copeland D, Lee A, Robinson SS. Goal-directed behavior in children with spina bifida. Dev Behav Pediatr. 1990; 11: 306-311.

48. Mammarella N, Cornoldi C, Donadello E. Visual but not spatial working memory deficit in children with spina bifida. Brain Cogn. 2003; 53: 311-314

49. Dennis M, Rogers T, Barnes MA. Children with spina bifida perceive visual illusions but not multi stable figures. Brain Cogn. 2001; 46: 108-113.

50. Jansen-Osmann P, Wiedenbauer G, Heil M. Spatial cognition and moto development: A study of children with spina bifida. Percep Mot Skill. 2008; 106: 436-446.

51. Leopold DA, Logotheitis NK. Multi stable phenomena: Changing views of perception. Trends Cogn Sci. 1999; 3: 254-264

52. Bundy AC, Lane SJ, Murray EA. Sensory Integration: Theory and Practice, $2^{\text {nd }}$ ed. Philadelphia, PA: FA Davis; 2002: 480.

53. Wiedenbauer G, Jansen-Osmann P. Spatial knowledge of children with spina bifida in a virtual large-scale space. Brain Cogn. 2006; 62: 120-127.

54. Rose BM, Holmbeck GN. Attention and executive functions in adolescents with spina bifida. J Pediatr Psychol. 2007; 32: 983-994.

55. Vinck A, Mullaart R, Rotteveel J, Maassen B. Neuropsychological assessment of attention in children with spina bifida. Cerebrospinal Fluid Res. 2009; 6: $6-13$
Phys Med Rehabil Int - Volume 4 Issue 1 - 2017 ISSN : 2471-0377 | www.austinpublishinggroup.com Martin et al. () All rights are reserved
Citation: Martin S and Kitzman P. Evidence of Cerebellar Dysfunction in Children with Myelomeningocele. Phys Med Rehabil Int. 2017; 4(1): 1109 